Congreso Latinoamericano de Ciencia Psicológica 2014. Asociación para el Avance de la Ciencia Psicológica, Buenos Aires, 2014.

\title{
Música y cognición performativa: legitimando el embodiment.
}

\section{Favio Shifres.}

Cita:

Favio Shifres (Octubre, 2014). Música y cognición performativa: legitimando el embodiment. Congreso Latinoamericano de Ciencia Psicológica 2014. Asociación para el Avance de la Ciencia Psicológica, Buenos Aires.

Dirección estable: https://www.aacademica.org/favio.shifres/193 ARK: https://n2t.net/ark:/13683/puga/eGv 
COGNICIÓN CORPOREIZADA

EMBODIED COGNITION

\title{
APLICACIONES DEL PARADIGMA CORPOREIZADO DE LA COGNICIÓN EN ESTUDIOS PSICOLÓGICOS

\author{
EMBODIED COGNITION AND EMBODIMENT. APPLICATIONS OF THE
} \\ EMBODIED COGNITION PARADIGM IN PSYCHOLOGICAL RESEARCH
}

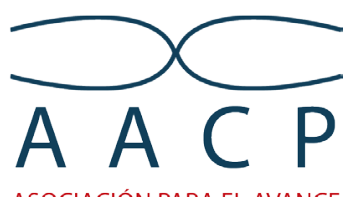

ASOCIACIÓN PARA EL AVANCE DE LA CIENCIA PSICOLÓGICA

(c) $2015 \cdot$ www.psiencia.org doi: $10.5872 /$ psiencia/7.1.021300

EJE ACADÉMICO ACADEMIC TRACK

\author{
Coordinador: Nicolás Alessandroni \\ nicoalessandroni@hotmail.com \\ Universidad Nacional de La Plata
}

\section{El embodiment en jaque: críticas, problemas y perspectivas actuales en el estudio de la cognición humana}

Nicolás Alessandroni

nicoalessandroni@hotmail.com

Universidad Nacional de La Plata

doi: $10.5872 /$ psiencia/7.1.021301

Resumen: En los últimos diez años, diversos autores han dirigido fuertes críticas a la Cognición Corporeizada por entender que no es posible concebirla como un verdadero programa de investigación del cual derivar hipótesis de trabajo legítimas. Estos autores proponen, en cambio, que el embodiment constituye una mera declaración de principios ontológicos metodológicamente incongruentes. Entendemos que estas críticas deben ser revisadas, toda vez que cuestionan las posibilidades explicativas y la plausibilidad psicológica de la Cognición Corporeizada, perspectiva que, por otro lado, ha logrado un nivel de impacto sumamente trascendente en la comunidad científica. DESARROLLO Se propone entonces una revisión inicial de los principios que sostienen el embodiment y un análisis de las críticas más importantes esgrimidas en los últimos años. Como una forma de enriquecer la discusión, se presenta un contrapunto de aportes provenientes del campo de la Psicología Cultural, en particular de aquellos autores que proponen un entrelazamiento entre las líneas ontogenética, filogenética y sociogenética como característica distintiva del desarrollo cognitivo humano. DISCUSIÓN Entendemos que las críticas realizadas al embodiment, sobre todo en su versión radical, son válidas e interesantes en tanto oportunidades para repensar la naturaleza de la cognición y su desarrollo. Se sostiene que al interior de la Psicología Cultural y otras áreas de desarrollo reciente como la Arqueología Cognitiva y la Neuroantropología es posible encontrar nuevos lineamientos, que podrían configurar un tipo de cognición más ecológica y psicológicamente plausible. Se plantea, por último, la necesidad de incorporar estas perspectivas al debate metodológico para la investigación en Ciencias Cognitivas.

\section{Música y cognición performativa: legitimando el embodiment}

\section{Favio Shifres}

favioshifres@gmail.com

Universidad Nacional de La Plata

doi: 10.5872/psiencia/7.1.021302

Resumen: El giro corporal en el estudio de la mente surgió de manera promisoria para superar escollos del cognitivismo clásico. No obstante, dicho giro es blanco de numerosas críticas. Habiendo el estudio de la experiencia musical puesto en tela de juicio muchos de las asunciones del paradigma clásico, debería resistir tales críticas. Sin embargo, ciertos supuestos musicológicos limitan el alcance de la música como dominio cognitivo impidiendo justipreciar el paradigma corporeizado para abordar la cognición humana incluyendo la experiencia musical y del pensamiento performativo. En tal sentido la dependencia de la psicología de la música respecto de la del lenguaje, parece ser clave. DESARROLLO: Tres tipos de prejuicios conceptuales imponen límites ontológicos a la música como dominio cognitivo: (i) el prejuicio estructural asume que la música compromete estructuras jerárquicas de constituyentes lingüísticos organizadas conforme gramáticas; (ii) el prejuicio funcional circunscribe las funciones de la música a su rol como sistema comunicacional; y (iii) el prejuicio modal confina los procesos cognitivos musicales a la operatoria con un imput sonoro. Existe, empero, numerosa evidencia para desarticular estos prejuicios.DISCUSIÓN: Clásicamente, la experiencia musical se ha estudiado con el auxilio de categorías conceptuales propias de la notación musical y la cultura musical alfabetizada de occidente. Por el contrario, el abordaje de la experiencia musical a partir de una 
redefinición de música con el aporte de conceptos de cuño corporeizado tales como Musicalidad Comunicativa y Formas Dinámicas de la Vitalidad, puede ser un campo fértil para el desarrollo de las propuestas corporeizadas.

\author{
Conciencia. Reconstruyendo el fenómeno \\ Sheilly Sánchez Rebolledo \\ shei.sanchez2013@gmail.com \\ Universidad de Buenos Aires
}

doi: 10.5872/psiencia/7.1.021303

Resumen: La concepción de sentido común acerca de la conciencia abarca un amplio espectro de fenómenos, desde los estados de vigilia hasta el pensamiento reflexivo. Sin embargo, desde la revolución de la ciencia cognitiva, se hizo necesario fraccionar el fenómeno en un conjunto de funciones y procesos que pudieran ser operacionalizados de manera independiente a los efectos de las masivas interacciones de la vida cotidiana. De modo que el fenómeno de la conciencia quedó desplazado como objeto de estudio en sí mismo, para ser abordado solo de manera indirecta, a partir de constructos tales como el de funciones ejecutivas, mecanismos atencionales o memoria autobiográfica (Funahashi, 2001) y el refinamiento de métodos y técnicas que ha permitido entender mejor la organización cerebral y sus interacciones. Mi objetivo es ofrecer una reconstrucción del concepto de conciencia sobre la base de los procesos, estructuras y constructos de la psicología actual, con el fin de rescatar el fenómeno como objeto directo de estudio. Esta reconstrucción intenta mostrar que las operaciones cognitivas superiores, como el pensar o recordar están ancladas en el procesamiento sensorial-perceptual y suponen más bien procesos de simulación (Damasio, 1989), en oposición a las concepciones clásicas que consideran el pensamiento en un medio simbólico abstracto. Uno de los alcances de la propuesta es que ofrece las condiciones que debería cumplir una teoría de las representaciones mentales, en favor de una teoría neoempirista de los conceptos (Prinz, 2002).

\title{
Cognición musical y embodiment: en la búsqueda de la experiencia perdida
}

Isabel Cecilia Martinez

isabelceciliamartinez@gmail.com

Universidad Nacional de La Plata

doi: 10.5872/psiencia/7.1.0201304

Resumen: La experiencia musical es un concepto cuya ontología resulta cuanto menos elusiva. La investigación en cognición musical de los últimos 50 años indagó los procesos que intervienen en la actividad musical desde los paradigmas representacionalista y computacional sin demostrar una preocupación explícita por definir sus alcances. El embodiment, al que hoy adscribe la psicología corporeizada de la música, propone una perspectiva más comprehensiva de experiencia, emergente del constructo mente-cuerpo-entorno y de su carácter multidimensional. Desarrollo. En este trabajo discutimos tópicos de la teoría del embodiment que, entendemos, pueden contribuir a caracterizar el concepto de experiencia musical. Se interrogarán la continuidad mente-cuerpo-entorno y la percepción-acción en la cognición musical, el movimiento como base para la construcción del significado corporeizado en música, y el sentido de sí mismo y del otro en la configuración de los perfiles dinámicos de la experiencia y la cognición musical sentida. Discusión. Se considera que los tópicos discutidos contribuyen a entender el modo en que la dimensión temporal, la atribución de intencionalidad a las acciones propias y de los otros, el desarrollo del pensamiento imaginativo y la construcción del significado se organizan en la experiencia musical. Nos interesa señalar que la revisión de estos aspectos del programa del embodiment constituye un esfuerzo intelectual por definir un concepto de experiencia musical que destaca su carácter multidimensional, corporeizado, intersubjetivo, distribuido y culturalmente situado.

\section{El rol de las simulaciones sensorio-motoras del dominio base de una metáfora conceptual en la interpretación de expresiones metafóricas}

Alejandra Martín, Juan Máximo Trench, Lucía Micaela Tavernini, Ricardo Adrián Minervino

micaelatavernini@yahoo.es

Universidad Nacional del Comahue, CONICET

doi: 10.5872/psiencia/7.1.021305

Resumen: Nos habíamos alejado de la primera década es una expresión metafórica (EM) en la que se habla del transcurso del tiempo en términos del movimiento corporal en el espacio. Lakoff y cols. (Lakoff, 2008) postularon que las personas emplean metáforas conceptuales (MCs) (e.g., la MC PERSONA EN 
MOVIMIENTO-TIEMPO, en la que PERSONA EN MOVIMIENTO es el dominio base y TIEMPO el dominio meta) a los fines de interpretar las EMs que se derivan de ellas, y que el trabajo de interpretación implica realizar simulaciones sensorio-motoras de la actividad referida por el dominio base (e.g., se simula la actividad de caminar hacia un objeto). Para poner a prueba esta última tesis, realizamos un experimento en el que se buscó determinar si la previa realización de una acción consistente con el dominio base de la MC PERSONA EN MOVIMIENTO-TIEMPO (caminar) facilita la lectura de EMs derivadas de esta MC, en comparación a la realización de una acción irrelevante (sentarse). Cada grupo de EMs se compuso de una EM sensorio-motora seguida de dos EMs de relleno. Mientras que los participantes de la condición consistente caminaron en línea recta hacia sucesivas computadoras en las que se detenían para leer grupos de EMs, aquellos pertenecientes a la condición neutral completaron su tarea en posición erguida frente a una computadora, sentándose y parándose nuevamente antes de leer cada grupo de EMs. Luego de la presentación de cada EM se efectuó una pregunta de comprensión sobre la misma. Se midieron los tiempos de lectura de las EMs. No se observaron diferencias en los tiempos de lectura entre la condición consistente y la condición neutral. Esto sugiere que la interpretación de EMs no conlleva la realización de simulaciones sensorio-motoras. Los resultados constituyen un desafío a la teoría corporeizada de la metáfora conceptual de Lakoff y cols. 